\title{
COMPACT DOMINATION FOR GROUPS DEFINABLE IN LINEAR O-MINIMAL STRUCTURES
}

\author{
PANTELIS E. ELEFTHERIOU
}

\begin{abstract}
We prove the Compact Domination Conjecture for groups definable in linear o-minimal structures. Namely, we show that every definably compact group $G$ definable in a saturated linear o-minimal expansion of an ordered group is compactly dominated by $\left(G / G^{00}, \mathbf{m}, \pi\right)$, where $\mathbf{m}$ is the Haar measure on $G / G^{00}$ and $\pi: G \rightarrow G / G^{00}$ is the canonical group homomorphism.
\end{abstract}

\section{INTRODUCTION}

The notion of compact domination arose in [HPP] in connection with Pillay's conjecture ([Pi2]) for groups definable in saturated o-minimal structures. Pillay's conjecture was proved in $[\mathrm{HPP}]$ for the case where the ambient o-minimal structure $\mathcal{M}$ expands an ordered field, in [ElSt] and [Ons] for the case where $\mathcal{M}$ is an ordered vector space over an ordered division ring, and in [Pet] for the intermediate case where $\mathcal{M}$ is semi-bounded. We state it here as a principle:

Pillay's Principle. Every definably compact group $G$ of dimension n, definable in a saturated o-minimal expansion $\mathcal{M}$ of an ordered group, has a smallest typedefinable subgroup $G^{00}$ of bounded index, and $G / G^{00}$, when equipped with the logic topology, is a compact Lie group of dimension $n$.

Compact domination is then intended to formalize the intuition that the canonical homomorphism $\pi: G \rightarrow G / G^{00}$ is a kind of intrinsic 'standard part map'. Recall the following definitions: A set $A \subseteq G / G^{00}$ is closed in the logic topology if $\pi^{-1}(A) \subseteq G$ is type-definable ([LaPi]). A subgroup $H$ of $G$ has bounded index if $|G / H|<|M|$.

When working over a saturated o-minimal expansion $\mathcal{M}$ of an ordered field, standard part maps have already appeared in the following two situations, among others. In [BO2, Definition 4.1], a standard part map is defined from the 'finite part' $\operatorname{Fin}\left(M^{n}\right)$ of $M^{n}$ onto $\mathbb{R}^{n}$, for $n \in \mathbb{N}_{+}$. In [PePi, Section 4], if $G(\mathbb{R})$ is a compact group of dimension $n$ definable in an o-minimal expansion $\mathcal{M}_{0}$ of $\mathbb{R}$, and $G$ is the realization of $G(\mathbb{R})$ in a saturated elementary extension $\mathcal{M}$ of $\mathcal{M}_{0}$, then a standard part map is defined from $G$ onto $G(\mathbb{R})$. In both cases, the standard part map st has the desired properties so that a notion of measure can be defined for the definable subsets of $\operatorname{Fin}\left(M^{n}\right)$ and $G$, respectively. Namely, if $\lambda$ denotes

Date: July 14, 2008 - Revised: March 24, 2009.

2000 Mathematics Subject Classification. 03C64, 22C05.

Key words and phrases. O-minimal structures, Definably compact groups, Compact domination.

Research supported by the FCT (Fundação para a Ciência e a Tecnologia) grant SFRH/BPD/35000/2007. 
the Lebesgue measure on $\mathbb{R}^{n}$, or the Haar measure on $G(\mathbb{R})$, respectively, then a definable set $X \subseteq \operatorname{Fin}\left(M^{n}\right)$, or $X \subseteq G$, respectively, is given measure $\lambda(\operatorname{st}(X))$.

We next recall some terminology from $[\mathrm{HPP}]$ and give the definition of compact domination.

Let $\mathcal{M}$ be a saturated structure. Unless specified otherwise, definability is meant in $\mathcal{M}$ with parameters. By a small set or a set of bounded cardinality we mean a set of cardinality less than $|M|$. By a type-definable set we mean an intersection of a small collection of definable sets. For a type-definable set $X$, by $\operatorname{Def}(X)$ we denote the set of all definable subsets of $X$.

Let $X$ be a set type-definable over $A$, and $C$ a compact Hausdorff space of bounded cardinality. A map $f: X \rightarrow C$ is called $A$-definable if for every closed set $C_{1} \subseteq C, f^{-1}\left(C_{1}\right) \subseteq X$ is type-definable over $A$. We say that $f$ is definable if it is $A$-definable for some set $A$.

A Keisler measure on $X$ is a finitely additive probability measure on $\operatorname{De} f(X)$, that is, a map $\mu: \operatorname{Def}(X) \rightarrow[0,1]$ such that $\mu(\emptyset)=0, \mu(X)=1$, and for $Y, Z \in \operatorname{Def}(X), \mu(Y \cup Z)=\mu(Y)+\mu(Z)-\mu(Y \cap Z)$.

Definition 1.1 ([HPP]). Suppose $X$ is a type-definable set, $C$ is a compact Hausdorff space of bounded cardinality equipped with a probability measure $\mu$, and $\sigma: X \rightarrow C$ is a definable surjective map. We say that $X$ is compactly dominated by $(C, \mu, \sigma)$ if for all $Y \in \operatorname{Def}(X)$,

$$
\mu\left(\left\{c \in C: \sigma^{-1}(c) \cap Y \neq \emptyset \text { and } \sigma^{-1}(c) \cap(X \backslash Y) \neq \emptyset\right\}\right)=0 .
$$

Let $G$ be a type-definable group. We say that $G$ is compactly dominated (as a group) if $G$ is compactly dominated by $(H, \mathbf{m}, \sigma)$, where $H$ is a compact Hausdorff group, $\mathbf{m}$ is the unique normalized $(\mathbf{m}(H)=1)$ Haar measure on $H$, and $\sigma$ is a group homomorphism.

When we work with a type-definable group, we always refer to compact domination in the group sense.

Fact 1.2 ([HPP], Proposition 9.3, Theorem 9.5). Let $G$ be a type-definable group which is compactly dominated by $(H, \mathbf{m}, \sigma)$. Then

(i) $G^{00}$ exists and equals $\operatorname{ker}(\sigma)$.

(ii) $G$ has a unique left (and right) invariant Keisler measure $\mu^{\prime}$, and it is given by: for all $X \in \operatorname{Def}(G), \mu^{\prime}(X)=\mathbf{m}(\sigma(X))$.

For the rest of this paper, let $\mathcal{M}$ be a sufficiently saturated o-minimal structure. If $G$ is a definable group, we denote by $m$ the unique normalized Haar measure on $G / G^{00}$, and by $\pi$ the definable group homomorphism from $G$ onto $G / G^{00}$.

Compact Domination Conjecture ([HPP $])$. Assume that $G$ is a definably compact definable group. Then $G$ is compactly dominated by $\left(G / G^{00}, \mathbf{m}, \pi\right)$.

Fact 1.3 ([HPP], Lemma 10.5). Suppose $G$ is a definably compact definable group of dimension $n$, such that, for all $X \in \operatorname{Def}(G)$,

$$
\operatorname{dim}(X)<n \Rightarrow \mathbf{m}(\pi(X))=0 .
$$

Then $G$ is compactly dominated by $\left(G / G^{00}, \mathbf{m}, \pi\right)$.

Let us note that (1) was a crucial property that implicitly held for st in place of $\pi$ in both accounts [BO2] and [PePi] mentioned above, for $X$ a definable subset 
of Fin $\left(M^{n}\right)$ in [BO2], and $X \in \operatorname{Def}(G)$ in [PePi], respectively. Additionally, st resembled $\pi$ in that a bounded set $X \subseteq \mathbb{R}^{n}$, or $X \subseteq G$, respectively, is closed if and only if $s t^{-1}(X)$ is type-definable.

By Fact 1.2(ii), Facts 1.4(i) and (ii)(a) below can be seen as a generalization of the existence of a measure from $[\mathrm{BO} 2]$ and $[\mathrm{PePi}]$, respectively.

Fact 1.4. (i) [HPP, Theorem 10.4] Assume $\mathcal{M}$ is a saturated o-minimal expansion of an ordered group. Then the unit $n$-cube $I^{n} \subseteq M^{n}$ is compactly dominated by $\left(I^{n}(\mathbb{R}), \lambda, s t\right)$, where $I^{n}(\mathbb{R}):=s t\left(I^{n}\right)$. ( $I^{n}$ and st are defined after a copy of $\mathbb{R}$ in $M$ is fixed; see [HPP, Section 10]).

(ii) [HPP, Theorem 10.7] Let $G$ be a definably compact definable group. Then $G$ is compactly dominated in either of the cases:

(a) G has a 'very good reduction'.

(b) $\operatorname{dim}(G)=1$.

In this paper, we give a positive answer to Compact Domination Conjecture in the case when $G$ is defined in a saturated linear o-minimal expansion $\mathcal{M}$ of an ordered group.

Definition 1.5 ([LoPe] $)$. An o-minimal expansion $\mathcal{M}=\langle M,+,<, 0, S\rangle$ of an ordered group is called linear if for every $\mathcal{M}$-definable function $f: A \subseteq M^{n} \rightarrow M$, there is a partition of $A$ into finitely many $A_{i}$, such that for each $i$, if $x, y, x+t, y+t \in$ $A_{i}$, then

$$
f(x+t)-f(x)=f(y+t)-f(y) .
$$

Theorem 1.6. Let $\mathcal{M}$ be a linear o-minimal expansion of an ordered group, and $G$ a definably compact group definable in $\mathcal{M}$. Then $G$ is compactly dominated by $\left(G / G^{00}, \mathbf{m}, \pi\right)$.

Remark 1.7. We may assume that $\mathcal{M}$ is an ordered vector space $\mathcal{N}$ over an ordered division ring. Indeed, by [LoPe], a linear o-minimal expansion $\mathcal{M}$ of an ordered group can be elementarily embedded into a reduct of an ordered vector space over an ordered division ring. Then $G$ (or, rather, $G(\mathcal{N})$ ) is definable in $\mathcal{N}$, and by [El, Section 5], $G_{\mathcal{M}}^{00}=G_{\mathcal{N}}^{00}$. Hence, to prove property (1) for $G$ it suffices to show that $G(\mathcal{N})$ remains definably compact with respect to definability in $\mathcal{N}$, and work in $\mathcal{N}$. To see that definable compactness is preserved under taking expansions, observe that $G$ can be written as $G=\bigcup_{i \in J} G_{i}$, where each $G_{i}$ is a closed subset of $G$ contained in one the charts of $G$ (see [BO1, Lemmas 10.4, 10.5], for example, where the authors work over a real closed field but their arguments go word-byword through in any o-minimal expansion of an ordered group). If $\phi_{i}: G_{i} \rightarrow M^{n}$ denotes the corresponding chart map, then $G$ is definably compact if and only if each $\phi_{i}\left(G_{i}\right)$ is closed and bounded. But the latter property is clearly preserved under taking expansions.

The strategy of our proof is (a) to define a standard part map $s t_{G}: G \rightarrow\left(S^{1}\right)^{n}$, $n=\operatorname{dim}(G)$, from $G$ to the real $n$-torus $\left(S^{1}\right)^{n}$ that 'resembles' $\pi: G \rightarrow G / G^{00}$, and (b) to show that $s t_{G}$ satisfies property (1).

In Section 2, we recall notation and several facts from [ElSt] where definable groups in an ordered vector space $\mathcal{M}$ over an ordered division ring $D$ were analyzed. It was shown there that every definably compact, definably connected definable group $G$ is definably isomorphic to a definable quotient group $U / L$, where $U$ is a $\bigvee$-definable subgroup of $\left\langle M^{n},+\right\rangle$, and $L$ is a lattice of rank $n$. In calculating the 
rank of $L$, a standard part map st $U \rightarrow \mathbb{R}^{n}$ was defined. Our main observation here is that, in a certain sense, $U$ corresponds to $\operatorname{Fin}\left(M^{n}\right)$ from [BO2], resulting to a correspondence between the associated standard maps as well.

In Section 3, we investigate properties of the standard part map st, making the correspondence with [BO2] more apparent.

Section 4 contains the main body of our proof. We use st to define a standard part map $s t_{G}: G \rightarrow\left(S^{1}\right)^{n}$ that resembles $\pi$ in that: $\operatorname{ker}\left(s t_{G}\right)=G^{00}$, and, for all $A \subseteq\left(S^{1}\right)^{n}, A$ is closed if and only if $s t_{G}^{-1}(A)$ is type-definable. We then show that $s t_{G}$ satisfies property (1). We conclude that $\pi$ satisfies (1), and, thus, $G$ is compactly dominated by $\left(G / G^{00}, \mathbf{m}, \pi\right)$.

Note. In the recent paper $[\mathrm{HP}]$, the Compact Domination Conjecture was proved in case $\mathcal{M}$ expands an ordered field and $G$ is abelian. The conjecture remains open in case $\mathcal{M}$ is semi-bounded.

Acknowledgements. The work presented here was carried out during my Ph.D. studies at the University of Notre Dame. I would like to thank my thesis supervisor Sergei Starchenko, as well as Ya'acov Peterzil for stimulating the subject. I would also like to thank the referee for the very careful reading and suggestions.

\section{Preliminaries}

We assume some familiarity with the basics of o-minimality; see [vdD] for a standard reference. In this section we fix notation and recall facts from [ElSt].

Let $\mathcal{M}=\langle M,+,<, \ldots\rangle$ be an o-minimal expansion of an ordered group. $M$ is equipped with the order topology. $M^{n}=\left\langle M^{n},+\right\rangle$ is the topological group whose group operation is defined point-wise, that has $0=(0, \ldots, 0)$ as its unit element, and whose topology is the product topology. If $L$ is a subgroup of $M^{n}$, we denote by $E_{L}$ the equivalence relation on $M^{n}$ induced by $L$, namely, $x E_{L} y \Leftrightarrow x-y \in L$. For $U \subseteq M^{n}$, we let $E_{L}^{U}:=E_{L} \uparrow_{U \times U}$ and $U / L:=U / E_{L}^{U}$. The elements of $U / L$ are denoted by $[x]_{L}^{U}, x \in U$. If $U \leqslant M^{n}$ is a subgroup of $M^{n}$, then it is a topological group equipped with the subspace topology. If, moreover, $L \leqslant U$, then $U / L=\left\langle U / L,+_{U / L},[0]_{L}^{U}\right\rangle$ is the quotient topological group, whose topological and group structure are both induced by the canonical surjection $q: U \rightarrow U / L$. If $S \subseteq U$ is a complete set of representatives for $E_{L}^{U}$ (that is, it contains exactly one representative for each equivalence class), then the bijection $f: U / L \rightarrow S$ defined by $[x]_{L}^{U} \mapsto x$ induces on $S$ a topological group structure $\left\langle S,{ }_{S}\right\rangle$ :

(i) $\forall x, y, z \in S, x+{ }_{S} y=z \Leftrightarrow[x]_{L}^{U}+_{U / L}[y]_{L}^{U}=[z]_{L}^{U} \Leftrightarrow(x+y) E_{L}^{U} z$, and

(ii) $A \subseteq S$ is open in the quotient topology if and only if $(f \circ q)^{-1}(A)$ is open in $U$.

$U / L$ is called a definable quotient group if there is a definable $S$ as above, such that $+_{S}$ is definable. In this case, we identify $U / L$ with $\left\langle S,{ }_{S}\right\rangle$.

An isomorphism between two topological groups is a group isomorphism which is also a homeomorphism.

The abelian subgroup of $M^{n}$ generated by the elements $v_{1}, \ldots, v_{m} \in M^{n}$ is denoted by $\mathbb{Z} v_{1}+\cdots+\mathbb{Z} v_{m}$. If $v_{1}, \ldots, v_{m}$ are $\mathbb{Z}$-linearly independent, then the free abelian subgroup $\mathbb{Z} v_{1}+\cdots+\mathbb{Z} v_{m}$ of $M^{n}$ is called a lattice of rank $m$.

For the rest of this paper let $\mathcal{M}=\left\langle M,+,<, 0,\{\lambda\}_{\lambda \in D}\right\rangle$ be a sufficiently saturated ordered vector space over an ordered division $\operatorname{ring} D=\langle D,+, \cdot,<$ 
$, 0,1\rangle$, and $G=\left\langle G, \oplus, e_{G}\right\rangle$ a definably connected, definably compact, definable group of dimension $n$. Unless specified otherwise, definability is meant in $\mathcal{M}$, possibly with parameters.

The group topology on $G$ is the $t$-topology from [Pi1]. The assumption of definable connectedness is at no loss of generality, again by [Pi1]. For the notion of definable compactness the reader is referred to [PeS]. By [vdD, Chapter 1, (7.6)], $\mathcal{M}$ is o-minimal. By [ElSt, Corollary 4.5], $G$ is abelian.

Definability in $\mathcal{M}$ was analyzed in [ElSt, Section 3] (see also [vdD, Chapter 1, $\S 7])$. A function $f: A \subseteq M^{m} \rightarrow M$ is called linear if it has form $f\left(x_{1}, \ldots, x_{n}\right)=$ $\lambda_{1} x_{1}+\cdots+\lambda_{n} x_{n}+a$, for some fixed $\lambda_{i} \in D$ and $a \in M$. The notion of a linear cell can then be defined similarly to that of a usual cell but using linear functions in place of definable continuous ones. The description of the definable sets is then given by the following.

Linear Cell Decomposition Theorem. Let $A \subseteq M^{n}$ and $f: A \rightarrow M$ be definable. Then there is a decomposition of $M^{n}$ that partitions $A$ into finitely many linear cells $A_{i}$, such that each $f \uparrow_{A_{i}}$ is linear.

$D=\langle D,+, \cdot,<, 0,1\rangle$ is a division ring and $\langle\mathbb{Q},+, \cdot,<, 0,1\rangle$ naturally embeds into $D$. A set $X \subseteq M^{n}$ is called convex if $\forall x, y \in X, \forall q \in \mathbb{Q} \cap[0,1], q x+(1-q) y \in X$. For $\lambda \in D,|\lambda|:=\max \{-\lambda, \lambda\}$, and for $x \in M,|x|:=\max \{-x, x\}$.

The main result from [ElSt] is the following.

Fact 2.1. $G$ is definably isomorphic to a definable quotient group $U / L$, where $U$ is a convex $\bigvee$-definable subgroup of $\left\langle M^{n},+\right\rangle$, and $L$ is a lattice of rank $n$.

For a definition of a $\bigvee$-definable group see [PeSt]. The $U$ mentioned in Fact 2.1 is a special case of a $\bigvee$-definable group, and its description is crucial for this paper. We next extract those ingredients from the proof of Fact 2.1 that will be used in our proof of compact domination.

If $\vec{\lambda}=\left(\lambda_{1}, \ldots, \lambda_{n}\right) \in D^{n}$ and $t \in M$, we denote $\vec{\lambda} t:=\left(\lambda_{1} t, \ldots, \lambda_{n} t\right)$. A set $H \subseteq G$ is called generic if finitely many $\oplus$-translates of it cover $G$. By the analysis in [ElSt], we may assume that $e_{G}=0$, and:

- $G$ contains a definable generic parallelogram:

$$
H:=\left\{\vec{\lambda}_{1} t_{1}+\cdots+\vec{\lambda}_{n} t_{n}:-e_{i}<t_{i}<e_{i}\right\},
$$

where $e_{1}, \ldots, e_{n} \in M$ are positive, and $\vec{\lambda}_{1}, \ldots, \vec{\lambda}_{n} \in D^{n}$.

- $U$ is the subgroup of $M^{n}$ generated by $H$ :

$$
U:=<H>=\bigcup_{k<\omega} \underbrace{H+\cdots+H}_{k-\text { times }} \leqslant M^{n} .
$$

- The following map $\phi: U \rightarrow G$ is a continuous, surjective group homomorphism: for all $x_{i} \in H$,

$$
\phi\left(x_{1}+\cdots+x_{k}\right)=x_{1} \oplus \cdots \oplus x_{k} .
$$

If we let $L:=\operatorname{ker}(\phi)$, then $U / L \cong G$ as abstract groups. In order to show that $L$ is a lattice of rank $n$, a standard part map st: $U \rightarrow \mathbb{R}^{n}$ was defined in [ElSt]. We recall the definition of $s t$ in Section 2.1 below. For the moment, we finish the 
description of the main ingredients of the proof of Fact 2.1. We recall that there is a positive $\Xi \in \mathbb{N}$, such that the set

$$
\Sigma:=H^{\Xi}=\left\{\vec{\lambda}_{1} t_{1}+\cdots+\vec{\lambda}_{n} t_{n}:-\Xi e_{i}<t_{i}<\Xi e_{i}\right\}
$$

contains a definable complete set $S$ of representatives for $E_{L}^{U}$. The restriction of $\phi$ on $S$ is then a definable isomorphism between the topological groups $\left\langle S,{ }_{S}\right\rangle$ and $G$. We may assume, up to definable isomorphism, that $G=S$ and

- $H \subseteq G \subseteq \Sigma \subseteq U$.

This assumption does not affect our proof of compact domination for $G$, since, easily, property (1), that we are aiming to show, remains invariant under definable isomorphisms.

Observe that for all $x \in H, \phi(x)=x$, and for all $x \in U,[\phi(x)]_{L}^{U}=[x]_{L}^{U}$.

2.1. A standard part map st $U \rightarrow \mathbb{R}^{n}$. Before we give the definition of the standard part map, we recall some basic facts and definitions from linear algebra over $D$.

We introduce some additional notation. If $\lambda=\left(\lambda^{1}, \ldots, \lambda^{n}\right) \in D^{n}$ and $\mu \in D$, we denote $\mu \lambda:=\left(\mu \lambda^{1}, \ldots, \mu \lambda^{n}\right)$ and $\lambda \mu:=\left(\lambda^{1} \mu, \ldots, \lambda^{n} \mu\right)$.

The elements $\lambda_{1}, \ldots, \lambda_{m} \in D^{n}$ are called left (right) D-independent if for all $\mu_{1}, \ldots, \mu_{m}$ in $D, \mu_{1} \lambda_{1}+\cdots+\mu_{m} \lambda_{m}=0\left(\lambda_{1} \mu_{1}+\cdots+\lambda_{m} \mu_{m}=0\right)$ implies $\mu_{1}=$ $\cdots=\mu_{m}=0$.

Let $A \in \mathbb{M}(n, D)$ be an $n \times n$ matrix with entries from $D$. The row rank of $A$ is the cardinality of a maximal left $D$-independent set of rows from $A$, and the column rank of $A$ is the cardinality of a maximal right $D$-independent set of columns from A.

Fact 2.2. (i) The row rank and the column rank of $A$ are equal. We refer to either of them as the rank of $A$.

(ii) The rank of $A$ is $n$ if and only if $A$ has an inverse.

Proof. (i) See [Jac, Chapter II, Theorem 9].

(ii) Similar to [Lang, Chapter IV, Theorem 2.2].

Now let

$$
H=\left\{\vec{\lambda}_{1} t_{1}+\cdots+\vec{\lambda}_{n} t_{n}:-e_{i}<t_{i}<e_{i}\right\}
$$

be the generic parallelogram of $G$, with $e_{1}, \ldots, e_{n} \in M$ positive, and $\vec{\lambda}_{1}, \ldots, \vec{\lambda}_{n} \in$ $D^{n}$, as described above. Since $H$ is generic, $\operatorname{dim}(H)=n$. Consider the following matrix with entries from $D$.

$$
A=\left(\begin{array}{lll}
\vec{\lambda}_{1} & \cdots & \vec{\lambda}_{n}
\end{array}\right)=\left(\begin{array}{ccc}
\lambda_{1}^{1} & \ldots & \lambda_{n}^{1} \\
\vdots & \ldots & \vdots \\
\lambda_{1}^{n} & \ldots & \lambda_{n}^{n}
\end{array}\right) .
$$

Claim 2.3. A has rank equal to $n$.

Proof. We show that $A$ has column rank $n$. Assume not. Without loss of generality, we may then assume that there are $\mu_{1}, \ldots, \mu_{n-1} \in D$ such that

$$
\vec{\lambda}_{n}=\vec{\lambda}_{1} \mu_{1}+\cdots+\vec{\lambda}_{n-1} \mu_{n-1} .
$$


But then

$$
\begin{aligned}
H & =\left\{\vec{\lambda}_{1} t_{1}+\cdots+\vec{\lambda}_{n} t_{n}:-e_{i}<t_{i}<e_{i}\right\} \\
& =\left\{\vec{\lambda}_{1} t_{1}+\cdots+\vec{\lambda}_{n-1} t_{n-1}+\left(\vec{\lambda}_{1} \mu_{1}+\cdots+\vec{\lambda}_{n-1} \mu_{n-1}\right) t_{n}:-e_{i}<t_{i}<e_{i}\right\} \\
& =\left\{\vec{\lambda}_{1}\left(t_{1}+\mu_{1} t_{n}\right) \cdots+\vec{\lambda}_{n-1}\left(t_{n-1}+\mu_{n-1} t_{n}\right):-e_{i}<t_{i}<e_{i}\right\},
\end{aligned}
$$

which clearly has dimension less than $n$, a contradiction.

Corollary 2.4. A is invertible.

Corollary 2.5. $\vec{\lambda}_{1}, \ldots, \vec{\lambda}_{n}$ are $M$-independent. That is, for all $t_{1}, \ldots, t_{n} \in M$,

$$
\vec{\lambda}_{1} t_{1}+\cdots+\vec{\lambda}_{n} t_{n}=0 \Rightarrow t_{1}=\cdots=t_{n}=0 .
$$

Proof. For any $t_{1}, \ldots, t_{n} \in M$, if $A\left(\begin{array}{c}t_{1} \\ \vdots \\ t_{n}\end{array}\right)=\left(\begin{array}{c}0 \\ \vdots \\ 0\end{array}\right)$, then $\left(\begin{array}{c}t_{1} \\ \vdots \\ t_{n}\end{array}\right)=A^{-1}\left(\begin{array}{c}0 \\ \vdots \\ 0\end{array}\right)=$ $\left(\begin{array}{c}0 \\ \vdots \\ 0\end{array}\right)$.

We now proceed to the definition of the standard part map st $U \rightarrow \mathbb{R}^{n}$. From the construction of $U$ given earlier, it is easy to see that every $u \in U$ has form

$$
u=\vec{\lambda}_{1} u_{1}+\cdots+\vec{\lambda}_{n} u_{n}
$$

with $u_{i} \in M$ and $-q e_{i}<u_{i}<q e_{i}$, for some $q \in \mathbb{Z}$. By Corollary 2.5, it follows that the $u_{i}$ 's in form (2) are unique. We define $s t: U \rightarrow \mathbb{R}^{n}$ as follows: for all $u \in U$, with form (2),

where for every $i$,

$$
s t(u)=\left(s t_{1}\left(u_{1}\right), \ldots, s t_{n}\left(u_{n}\right)\right)
$$

$$
s t_{i}\left(u_{i}\right)=\sup \left\{q \in \mathbb{Q}: q e_{i}<u_{i}\right\} .
$$

It can be checked that $s t$ is a surjective group homomorphism.

The notation ' $\vec{\lambda}_{1}, \ldots, \vec{\lambda}_{n}, H, U, \phi, L, \Xi, \Sigma, s t$ ' is fixed for the rest of this paper.

2.2. On Pillay's Principle. $G^{00}$ is defined as follows. For $k \in \mathbb{N}$, we define $H_{k}$ inductively: $H_{0}=H$, and $H_{k+1}=\frac{1}{2} H_{k}$. Then it is not hard to see that $\bigcap_{k<\omega} H_{k}$ is a torsion-free, type-definable subgroup of $G$ of bounded index, and therefore, by $[\mathrm{BOPP}]$ :

$$
G^{00}=\bigcap_{k<\omega} H_{k}
$$

Note that $G^{00}$ is open in $M^{n}$.

Lemma 2.6. $\operatorname{ker}(s t)=G^{00}$.

Proof. For all $x \in G$,

$$
\begin{aligned}
x \in G^{00} & \Leftrightarrow x=\vec{\lambda}_{1} h_{1}+\cdots+\vec{\lambda}_{n} h_{n}, \text { for some } h_{i} \text { with } \forall k \in \mathbb{N},-\frac{1}{2^{k}} e_{i}<h_{i}<\frac{1}{2^{k}} e_{i}, \\
& \Leftrightarrow x=\vec{\lambda}_{1} h_{1}+\cdots+\vec{\lambda}_{n} h_{n}, \text { for some } h_{i} \text { with } \forall q \in \mathbb{Q}^{>0},-q e_{i}<h_{i}<q e_{i}, \\
& \Leftrightarrow s t(x)=0 .
\end{aligned}
$$




\section{Properties of $s t: U \rightarrow \mathbb{R}^{n}$}

The definition of a box in $U$ given below is in analogy with the definition from [BO2] of a box in cartesian powers of an o-minimal expansion of an ordered field.

Definition 3.1. Let $n \in \mathbb{N}, n>0$. A box (of dimension $n$ ) is a subset of $U$ of the form

for some $p_{i}, q_{i} \in \mathbb{Q}$, with $p_{i} \leq q_{i}$.

$$
B=\left\{\vec{\lambda}_{1} t_{1}+\cdots+\vec{\lambda}_{n} t_{n}: p_{i} e_{i} \leq t_{i} \leq q_{i} e_{i}\right\},
$$

A real box (of dimension $n$ ) is a subset of $\mathbb{R}^{n}$ of the form

$$
C=\left[k_{1}, l_{1}\right] \times \cdots \times\left[k_{n}, l_{n}\right]
$$

for some $k_{i}, l_{i} \in \mathbb{Q}$, with $k_{i} \leq l_{i}$.

If $B$ is a box as above, then $B^{\mathbb{R}}$ denotes the real box defined by $k_{i}=p_{i}$ and $l_{i}=q_{i}$, for $i=1, \ldots, n$.

Let $k \in \mathbb{N}, k>0$. A real $\frac{1}{k}$-box of dimension $n$ is a real box $\subseteq \mathbb{R}^{n}$ of the form

$$
C=\left[\frac{k_{1}}{k}, \frac{k_{1}+1}{k}\right] \times \cdots \times\left[\frac{k_{n}}{k}, \frac{k_{n}+1}{k}\right],
$$

for some $k_{i} \in \mathbb{Z}$.

The base of such a $C$ is the real $\frac{1}{k}$-box of dimension $n-1$ in $\mathbb{R}^{n-1}$ :

$$
\left[\frac{k_{1}}{k}, \frac{k_{1}+1}{k}\right] \times \cdots \times\left[\frac{k_{n-1}}{k}, \frac{k_{n-1}+1}{k}\right] .
$$

Remark 3.2. It is easy to see that, for all $x \in U$ and $y \in \mathbb{R}^{n}$,

$$
s t(x)=y \Leftrightarrow \text { for every box } B \subseteq U, x \in B \text { implies } y \in B^{\mathbb{R}} .
$$

The proof of the following lemma is almost word-by-word the one of [BO2, Proposition 4.2].

Lemma 3.3. For every box $B \subseteq U$, st $(B)=B^{\mathbb{R}}$.

Proof. The inclusion $s t(B) \subseteq B^{\mathbb{R}}$ is by Remark 3.2. For the equality, let $y \in B^{\mathbb{R}}$. We write $\{y\}=\bigcap_{i \in \mathbb{N}} B_{i}^{\mathbb{R}}$, where $\left\{B_{i}^{\mathbb{R}}: i \in \mathbb{N}\right\}$ is an enumeration of all real boxes containing $y$. The set of all formulas $x \in B_{i}$ is a type in $M$ which must be realized by some element $x \in U$. For this $x$, we have $s t(x)=y$ and $x \in B$.

The properties listed below will be used in the sequel. For $X \subseteq M^{n}$, we denote by $\bar{X}$ the closure of $X$ in $M^{n}$.

Lemma 3.4. (i) For all $X_{1}, X_{2} \subseteq U$, st $\left(X_{1} \cup X_{2}\right)=\operatorname{st}\left(X_{1}\right) \cup \operatorname{st}\left(X_{2}\right)$.

(ii) For all $X \subseteq U$, st ${ }^{-1}(\operatorname{st}(X))=X+G^{00}$.

(iii) For all $X \subseteq U$, st $(\bar{X})=\operatorname{st}(X)$.

(iv) For all $X \subseteq U, X+G^{00}=\bar{X}+G^{00}$.

(v) $A$ bounded set $A \subseteq \mathbb{R}^{n}$ is closed if and only if st ${ }^{-1}(A)$ is type-definable.

(vi) For all definable $X \subseteq U$, st $(X)$ is closed.

Proof. (ii) $\forall y \in U$,

$$
y \in s t^{-1}(s t(X)) \Leftrightarrow \exists x \in X, s t(x)=s t(y) \Leftrightarrow y \in X+G^{00} .
$$

(iii) For the non-trivial inclusion $(\subseteq)$, let $x \in \bar{X}$. We need to find $x^{\prime} \in X$, such that $\operatorname{st}\left(x^{\prime}\right)=\operatorname{st}(x)$. Since $G^{00}$ is open and $x \in \bar{X},\left(x+G^{00}\right) \cap X \neq \emptyset$. We can take $x^{\prime}$ to be any element in $\left(x+G^{00}\right) \cap X$. 
(iv) By (ii) and (iii), $X+G^{00}=s t^{-1}(s t(X))=s t^{-1}(s t(\bar{X}))=\bar{X}+G^{00}$.

(v) The proof is almost word-by-word the one of [BO2, Proposition 5.4]. Note that by (ii) and Lemma 3.3, for every box $B \subseteq U$, st $t^{-1}\left(B^{\mathbb{R}}\right)=B+G^{00}$.

Let $A \subseteq \mathbb{R}^{n}$ be bounded.

For the left-to-right direction, if $A$ is closed, then $A=\bigcap_{i \in \mathbb{N}} \bigcup_{j \in J_{i}} B_{i j}^{\mathbb{R}}$ is the intersection of a countable family of unions of finitely many real boxes. Thus, $s t^{-1}(A)=\left\{x: \bigwedge_{i \in \mathbb{N}} \bigvee_{j \in J_{i}}\left(x \in B_{i j}+G^{00}\right)\right\}$ is type-definable.

For the right-to-left direction, let $s t^{-1}(A)$ be type-definable, say $s t^{-1}(A)=\{x$ : $\left.\bigwedge_{i \in I}\left(x \in X_{i}\right)\right\}$, where each $X_{i}$ is definable. To show that $A$ is closed, let $y \in \bar{A}$. We show that $y \in A=s t\left(s t^{-1}(A)\right)$. We need to find an $x \in \bigcap_{i \in I} X_{i}$ such that $s t(x)=y$. It suffices to show that the set $\bigcap_{i \in I} X_{i} \cap \bigcap_{j \in \mathbb{N}} B_{j}$ is non-empty, where $\left\{B_{j}: j \in \mathbb{N}\right\}$ is an enumeration of all boxes $B$ with $y \in \operatorname{Int}\left(B^{\mathbb{R}}\right)$. By compactness, it suffices to show that the set $P=\bigcap_{i \in I} X_{i} \cap B$ is non-empty, where $B$ is any box with $y \in \operatorname{Int}\left(B^{\mathbb{R}}\right)$. But, since $y \in \bar{A}$, there is $y^{\prime} \in \operatorname{Int}\left(B^{\mathbb{R}}\right) \cap A \neq \emptyset$. Thus, there is $x^{\prime} \in B$, such that $s t\left(x^{\prime}\right)=y^{\prime} \in B^{\mathbb{R}} \cap A$. This $x^{\prime}$ belongs to $P$.

(vi) Observe that the boundedness of $A \subseteq \mathbb{R}^{n}$ in (v) was only used in the leftto-right direction. Now, let $X \subseteq U$. By (ii), $s t^{-1}(s t(X))=X+G^{00}$, so if $X$ is definable, then $s t^{-1}(s t(X))$ is type-definable and we can apply (v), right-to-left.

\section{4. $G$ is COMPACTLY DOMinAted}

We start with defining a standard part map for $G$. Recall $L=\operatorname{ker}(\phi)$ has rank $n$. Let $L=\mathbb{Z} v_{1}+\cdots+\mathbb{Z} v_{n} \leqslant U \leqslant M^{n}$.

Claim 4.1. $s t(L) \subseteq \mathbb{R}^{n}$ is a discrete lattice of rank $n$. Therefore, $\mathbb{R}^{n} / \operatorname{st}(L)$ is isomorphic to the real $n$-torus $\left(S^{1}\right)^{n}$.

Proof. $\operatorname{st}(L)$ is discrete: since for all $x \in H, \phi(x)=x$, we have $\operatorname{ker}(\phi) \cap H=\{0\}$. Hence the interior of $s t(H)$ is an open neighborhood of 0 that contains no other elements of $s t(L)$.

$\operatorname{st}(L)$ has rank $n$ : clearly, st $(L)$ has rank at most $n$. Now assume, towards a contradiction, that for some $l_{1}, \ldots, l_{n} \in \mathbb{Z}$, not all zero, $l_{1} s t\left(v_{1}\right)+\cdots+l_{n} s t\left(v_{n}\right)=0$. Since $s t: U \rightarrow \mathbb{R}^{n}$ is a group homomorphism, st $\left(l_{1} v_{1}+\cdots+l_{n} v_{n}\right)=0$. Thus, $l_{1} v_{1}+\cdots+l_{n} v_{n} \in G^{00} \subseteq H$. Since $\operatorname{ker}(\phi) \cap H=\{0\}$, we have $l_{1} v_{1}+\cdots+l_{n} v_{n}=0$, contradicting the fact that $L$ has rank $n$.

Let $q$ denote the canonical homomorphism from $\mathbb{R}^{n}$ onto $\mathbb{R}^{n} / \operatorname{st}(L)$. let

We define a standard part map $s t_{G}: G \rightarrow \mathbb{R}^{n} / \operatorname{st}(L)$ as follows. For all $x \in G$,

$$
s t_{G}(x)=q(s t(x))=[s t(x)]_{s t(L)}^{\mathbb{R}^{n}} .
$$

Since $s t$ is a group homomorphism, so is $s t_{G}$. Indeed, for all $x, y \in G$, we have $x \oplus y=\phi(x+y) \in x+y+L$, and

$$
\begin{aligned}
& (x \oplus y)-(x+y) \in L \Rightarrow s t(x \oplus y)-(s t(x)+s t(y)) \in \operatorname{st}(L) \\
& \Leftrightarrow[s t(x \oplus y)]_{s t(L)}^{\mathbb{R}^{n}}=[s t(x)+s t(y)]_{s t(L)}^{\mathbb{R}^{n}}=[s t(x)]_{s t(L)}^{\mathbb{R}^{n}}+\mathbb{R}^{n} / s t(L)[s t(y)]_{s t(L)}^{\mathbb{R}^{n}} \\
& \Leftrightarrow s t_{G}(x \oplus y)=s t_{G}(x)++_{\mathbb{R}^{n}} / s t(L) s t_{G}(y) .
\end{aligned}
$$

Also, $\operatorname{ker}\left(s t_{G}\right)=G^{00}$. Indeed, for all $x \in G, s t_{G}(x)=[0]_{s t(L)}^{\mathbb{R}^{n}} \Leftrightarrow \operatorname{st}(x) \in \operatorname{st}(L) \Leftrightarrow$ $x \in\left(G^{00}+L\right) \cap G=G^{00}$, since $G^{00} \subseteq G$ and $G \cap L=\{0\}$. 
Lemma 4.2. The following diagram commutes,

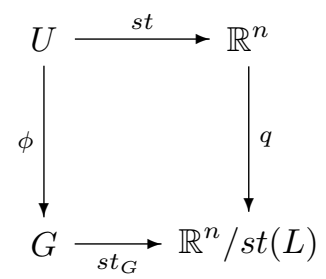

namely, $q \circ s t=s t_{G} \circ \phi$. Thus, in particular, for all $A \subseteq \mathbb{R}^{n} / \operatorname{st}(L)$,

$$
s t^{-1}\left(q^{-1}(A)\right)=\phi^{-1}\left(s t_{G}^{-1}(A)\right) .
$$

Proof. First, notice that for all $x, y \in U$, if $[x]_{L}^{U}=[y]_{L}^{U}$, then $[\operatorname{st}(x)]_{s t(L)}^{\mathbb{R}^{n}}=$ $[s t(y)]_{s t(L)}^{\mathbb{R}^{n}}$. This is because st is a group homomorphism:

$$
x-y \in L \Rightarrow s t(x-y) \in s t(L) \Leftrightarrow s t(x)-s t(y) \in s t(L) .
$$

Now, let $x \in U$. On the one hand, we have $q(\operatorname{st}(x))=[s t(x)]_{s t(L)}^{\mathbb{R}^{n}}$. On the other hand, $\phi(x) \in G$ with $[x]_{L}^{U}=[\phi(x)]_{L}^{U}$, and, thus, $[s t(x)]_{s t(L)}^{\mathbb{R}^{n}}=[s t(\phi(x))]_{s t(L)}^{\mathbb{R}^{n}}=$ $s t_{G}(\phi(x))$. Hence, $q(s t(x))=s t_{G}(\phi(x))$.

Lemma 4.3. $\bar{\Sigma}^{\mathbb{R}}=s t(\Sigma) \subseteq \mathbb{R}^{n}$ contains a set of representatives for $E_{s t(L)}^{\mathbb{R}^{n}}$. Thus, for all $A \subseteq \mathbb{R}^{n} / \operatorname{st}(L), A$ is closed (in the quotient topology) if and only if $\bar{\Sigma}^{\mathbb{R}} \cap$ $q^{-1}(A) \subseteq \mathbb{R}^{n}$ is closed.

Proof. Let $y \in \mathbb{R}^{n}$. Pick $x \in U$ such that $s t(x)=y$. Let $g \in G$ such that $g-x \in L$. Then $s t(g)-y=s t(g-x) \in \operatorname{st}(L)$. But $s t(g) \in s t(G) \subseteq \bar{\Sigma}^{\mathbb{R}}$.

For the second claim, let $A \subseteq \mathbb{R}^{n} / \operatorname{st}(L)$. If $A$ is closed, then $q^{-1}(A) \subseteq \mathbb{R}^{n}$ is closed, and, thus, $\bar{\Sigma}^{\mathbb{R}} \cap q^{-1}(A)$ is closed. Conversely, if $\bar{\Sigma}^{\mathbb{R}} \cap q^{-1}(A)$ is closed, then $\left(\bar{\Sigma}^{\mathbb{R}} \cap q^{-1}(A)\right)+\operatorname{st}(L)$ is closed. But since $\bar{\Sigma}^{\mathbb{R}}$ contains a set $S$ of representatives for $E_{s t(L)}^{\mathbb{R}^{n}}$, we have

$$
q^{-1}(A)=\left(S \cap q^{-1}(A)\right)+s t(L) \subseteq\left(\bar{\Sigma}^{\mathbb{R}} \cap q^{-1}(A)\right)+s t(L) \subseteq q^{-1}(A),
$$

that is, $q^{-1}(A)=\left(\bar{\Sigma}^{\mathbb{R}} \cap q^{-1}(A)\right)+s t(L)$ is closed, and, thus, $A$ is closed.

By Pillay's Principle, $G / G^{00}$ (equipped with the logic topology) is a connected, compact, abelian Lie group of dimension $n$ and, therefore, it is isomorphic to $\mathbb{R}^{n} / s t(L)$. The following lemma implies that the alleged isomorphism is indeed witnessed by the map $f: G / G^{00} \rightarrow \mathbb{R}^{n} / \operatorname{st}(L)$ defined by:

$$
f: x \oplus G^{00} \mapsto s t_{G}(x) .
$$

(As a side remark, $f$ is not an isomorphism if seen as the induced quotient map where $G / G^{00}$ has the quotient topology; that would be the case if $f$ were open. In any case, such an $f$ would not be what we need here, since the logic topology on $G / G^{00}$ is different from the quotient one, [Pi2, Remark 3.3].) We denote by $\pi: G \rightarrow G / G^{00}$ the canonical surjective homomorphism; then $f$ is by definition the 
unique map that makes the following diagram commute:

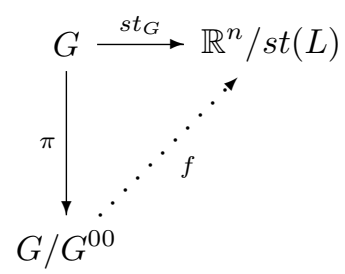

Remark 4.4. The map $f: G / G^{00} \rightarrow \mathbb{R}^{n} / \operatorname{st}(L)$, with $x \oplus G^{00} \mapsto s t_{G}(x)=[s t(x)]_{s t(L)}^{\mathbb{R}^{n}}$ is a group isomorphism.

Proof. $f$ is well-defined and it is injective, since for all $x, y \in G$,

$$
x \oplus G^{00}=y \oplus G^{00} \Leftrightarrow x \ominus y \in G^{00} \Leftrightarrow s t_{G}(x \ominus y)=0 \Leftrightarrow s t_{G}(x)=s t_{G}(y) .
$$

Easily, $f$ is a group homomorphism, since $s t_{G}$ is. That it is surjective, is essentially Lemma 4.3: given $\operatorname{st}(z) \in \mathbb{R}^{n}$, we can find $g \in G$, such that $\operatorname{st}(g)-\operatorname{st}(z) \in \operatorname{st}(L)$.

Lemma 4.5. For all $A \subseteq \mathbb{R}^{n} / \operatorname{st}(L), A$ is closed if and only if $t_{G}^{-1}(A)$ is typedefinable. Thus, $f$ is an isomorphism between topological groups.

Proof. By Lemma 3.4(v), a bounded set $A \subseteq \mathbb{R}^{n}$ is closed if and only if $s t^{-1}(A)$ is type-definable. Now, let $A \subseteq \mathbb{R}^{n} / \operatorname{st}(L)$. Then, $A$ is closed

if and only if (Lemma 4.3) $\bar{\Sigma}^{\mathbb{R}} \cap q^{-1}(A) \subseteq \mathbb{R}^{n}$ is closed

if and only if $s t^{-1}\left(\bar{\Sigma}^{\mathbb{R}} \cap q^{-1}(A)\right)$ is type-definable

if and only if $s t^{-1}\left(\bar{\Sigma}^{\mathbb{R}}\right) \cap s t^{-1}\left(q^{-1}(A)\right)$ is type-definable

if and only if $\left(\Sigma+G^{00}\right) \cap s t^{-1}\left(q^{-1}(A)\right)$ is type-definable

if and only if $\phi\left(\left(\Sigma+G^{00}\right) \cap s t^{-1}\left(q^{-1}(A)\right)\right)$ is type-definable,

where the last equivalence is because $\phi_{\uparrow \Sigma+G^{00}}$ is type-definable. Indeed, the 'only if' part is clear, whereas for the 'if' part, let $B:=\left(\Sigma+G^{00}\right) \cap s t^{-1}\left(q^{-1}(A)\right)$. We show that if $\phi(B)$ is type-definable, then $B$ is as well. To this end, we show that

$$
B=\left\{y \in \Sigma+G^{00}: \phi_{\left\lceil\Sigma+G^{00}\right.}(y) \in \phi_{\uparrow \Sigma+G^{00}}(B)\right\} .
$$

To see this, let $y \in \Sigma+G^{00}$ be such that $\phi(y)=\phi(b)$, for some $b \in B$. Then

$$
q(s t(y))=s t_{G}(\phi(y))=s t_{G}(\phi(b))=q(s t(b)) \in A,
$$

by Lemma 4.2 , showing that $y \in s t^{-1}\left(q^{-1}(A)\right)$, and, thus, $y \in B$. This completes the proof of the last 'if and only if'.

Therefore, we will be done with the first statement of the lemma if we show that

$$
\phi\left(\left(\Sigma+G^{00}\right) \cap s t^{-1}\left(q^{-1}(A)\right)\right)=s t_{G}^{-1}(A) .
$$

First, we observe that

$$
\phi\left(\left(\Sigma+G^{00}\right) \cap s t^{-1}\left(q^{-1}(A)\right)\right)=\phi\left(s t^{-1}\left(q^{-1}(A)\right)\right) .
$$

Indeed, for the non-trivial inclusion $(\supseteq)$, let $\phi(x) \in \phi\left(s t^{-1}\left(q^{-1}(A)\right)\right)$, for some $x \in$ $U$ with $q(\operatorname{st}(x)) \in A$. Then we find $y \in \Sigma+G^{00}$ with $\phi(x)=\phi(y)$ and $q(\operatorname{st}(y)) \in A$, as follows. Let $y \in \Sigma$ be such that $x-y \in L$. Then, on the one hand, $\phi(x)=\phi(y)$, and on the other, st $(x)-s t(y) \in \operatorname{st}(L)$ and, thus, $q(s t(y))=q(\operatorname{st}(x)) \in A$. 
Now, by Lemma $4.2, s t^{-1}\left(q^{-1}(A)\right)=\phi^{-1}\left(s t_{G}^{-1}(A)\right)$. Since $\phi$ is onto,

$$
\phi\left(s t^{-1}\left(q^{-1}(A)\right)\right)=\phi\left(\phi^{-1}\left(s t_{G}^{-1}(A)\right)\right)=s t_{G}^{-1}(A) .
$$

This proves (3).

For the second statement, by Remark 4.4, it remains to show that $f$ is a homeomorphism. We note that this can also be obtained by [HPP, Remark 2.3(i)] and the first part of this lemma; we provide a direct proof here (still using the first part). Let $A \subseteq \mathbb{R}^{n} / s t(L)$. We show that $A$ is closed if and only if $f^{-1}(A)=\pi\left(s t_{G}^{-1}(A)\right)$ is closed (in the logic topology). By the first part of this lemma, $A$ is closed if and only if $s t_{G}^{-1}(A)$ is type-definable. Since $s t_{G}^{-1}(A)=s t_{G}^{-1}(A)+G^{00}$, we get

$$
\pi^{-1}\left(\pi\left(s t_{G}^{-1}(A)\right)\right)=\pi^{-1}\left(\pi\left(s t_{G}^{-1}(A)+G^{00}\right)\right)=s t_{G}^{-1}(A)+G^{00}=s t_{G}^{-1}(A)
$$

and, therefore, $s t_{G}^{-1}(A)$ is type-definable if and only if $\pi\left(s t_{G}^{-1}(A)\right)$ is closed in the logic topology.

The compact Lie group $G / G^{00}$ has a unique normalized Haar measure $\mathbf{m}$. Thus, if $\mathbf{m}^{\prime}$ is a Haar measure on $\mathbb{R}^{n} / s t(L)$, then there is a positive $r \in \mathbb{R}$, such that for all $A \subseteq G / G^{00}, A$ is $\mathbf{m}$-measurable if and only if $f(A)$ is $\mathbf{m}^{\prime}$-measurable, and, if they are, then

$$
\mathbf{m}(A)=r \mathbf{m}^{\prime}(f(A)) .
$$

Since for all $X \subseteq G, f(\pi(X))=s t_{G}(X)$, in order to show property (1) from Introduction for $\pi$ it is thus equivalent to show it for $s t_{G}$, that is, to show, for all definable $X \subseteq G$,

$$
\operatorname{dim}(X)<n \Rightarrow \mathbf{m}^{\prime}\left(s t_{G}(X)\right)=0 .
$$

On the other hand, a Haar measure $\mathbf{m}^{\prime}$ on $\mathbb{R}^{n} / s t(L)$ can be defined using the Lebesgue measure $\lambda$ on $\mathbb{R}^{n}$, as follows. First, let $S \subseteq \mathbb{R}^{n}$ be a fundamental domain for $E_{s t(L)}^{\mathbb{R}^{n}}$ which is Lebesgue measurable. For example, if $A_{1}, \ldots, A_{n} \subseteq \mathbb{R}^{n}$ denote the line segments from 0 to the generators of $s t(L)$, excluding the generators, we may let $S=A_{1}+\cdots+A_{n}$. Now, for $X \subseteq \mathbb{R}^{n} / \operatorname{st}(L)$, let

$$
\mathbf{m}^{\prime}(X):=\lambda\left(S \cap q^{-1}(X)\right),
$$

assuming that $S \cap q^{-1}(X)$ is a Lebesgue measurable subset of $\mathbb{R}^{n}$. It is an easy classical fact that, if $A \subseteq \mathbb{R}^{n}$ is Lebesgue measurable, then for all $B \subseteq S$ with $q(B)=q(A), B$ is Lebesgue measurable and $\lambda(B) \leq \lambda(A)$. Therefore, for every $X \subseteq G$, such that $s t(X)$ is Lebesgue measurable, $S \cap q^{-1}\left(s t_{G}(X)\right)$ is Lebesgue measurable and

$$
\mathbf{m}^{\prime}\left(s t_{G}(X)\right) \leq \lambda(\operatorname{st}(X)) .
$$

It follows that in order to show (5), it suffices to show the following.

Lemma 4.6. Let $X \subseteq U$ be definable. If $\operatorname{dim}(X)<n$, then $\lambda(\operatorname{st}(X))=0$.

Proof. By Lemma 3.4(vi), st $(X)$ is closed and hence Lebesgue measurable.

If $n=1$, then $X$ and $\operatorname{st}(X)$ are finite, hence $\lambda(s t(X))=0$. Let $n>1$. We may assume that $\operatorname{dim}(X)=n-1$. By the Linear Cell Decomposition Theorem and Lemma 3.4(i), we may assume that $X$ is the graph of some linear function $f: C \rightarrow M$, where $C$ is a definable subset of $M^{n-1}$, after perhaps rearranging 
coordinates.

Claim 1. There are $\xi_{1}, \ldots, \xi_{n} \in D$, not all zero, and $c \in M$, such that for all $t_{1}, \ldots, t_{n} \in M$, if $\vec{\lambda}_{1} t_{1}+\cdots+\vec{\lambda}_{n} t_{n} \in X$, then $\xi_{1} t_{1}+\cdots+\xi_{n} t_{n}+c=0$.

Proof of Claim 1. Assume that for all $x=\left(x_{1}, \ldots, x_{n-1}\right) \in C$,

$$
f(x)=\mu_{1} x_{1}+\cdots+\mu_{n-1} x_{n-1}+c,
$$

for some fixed $\mu_{i} \in D$ and $c \in M$. Assume also that for $i=1, \ldots, n$,

$$
\vec{\lambda}_{i}=\left(\begin{array}{c}
\lambda_{i}^{1} \\
\vdots \\
\lambda_{i}^{n}
\end{array}\right) \in D^{n} .
$$

For each $i=1, \ldots, n$, we set

$$
\xi_{i}:=\mu_{1} \lambda_{i}^{1}+\cdots+\mu_{n-1} \lambda_{i}^{n-1}-\lambda_{i}^{n} .
$$

Consider any $t_{1}, \ldots, t_{n} \in M$ with $\lambda_{1} t_{1}+\ldots \lambda_{n} t_{n} \in X$, and let $x=\left(x_{1}, \ldots, x_{n-1}\right) \in$ $C$ be such that $(x, f(x))=\lambda_{1} t_{1}+\ldots \lambda_{n} t_{n}$. Then:

$$
\begin{aligned}
x_{1} & =\lambda_{1}^{1} t_{1}+\cdots+\lambda_{n}^{1} t_{n} \\
& \vdots \\
x_{n-1} & =\lambda_{1}^{n-1} t_{1}+\cdots+\lambda_{n}^{n-1} t_{n} \\
\mu_{1} x_{1}+\cdots+\mu_{n-1} x_{n-1}+c & =\lambda_{1}^{n} t_{1}+\cdots+\lambda_{n}^{n} t_{n}
\end{aligned}
$$

Substituting the first $n-1$ equations into the last one, we obtain:

$$
\left(\mu_{1} \lambda_{1}^{1}+\cdots+\mu_{n-1} \lambda_{1}^{n-1}-\lambda_{1}^{n}\right) t_{1}+\cdots+\left(\mu_{1} \lambda_{n}^{1}+\cdots+\mu_{n-1} \lambda_{n}^{n-1}-\lambda_{n}^{n}\right) t_{n}+c=0,
$$

that is,

$$
\xi_{1} t_{1}+\cdots+\xi_{n} t_{n}+c=0 .
$$

So, to finish the proof of Claim 1 , we need to see that not all $\xi_{i}$ 's are zero. It is enough to see that the matrix

$$
A=\left(\begin{array}{ccc}
\lambda_{1}^{1} & \ldots & \lambda_{n}^{1} \\
\vdots & \ldots & \vdots \\
\lambda_{1}^{n} & \ldots & \lambda_{n}^{n}
\end{array}\right) .
$$

has row rank $n$. But this is by Claim 2.3.

Without loss of generality, we may assume that there are $\nu_{1}, \ldots, \nu_{n-1} \in D$, $\left|\nu_{i}\right| \leq 1$, and $d \in M$, such that for all $t_{1}, \ldots, t_{n} \in M$,

$$
\vec{\lambda}_{1} t_{1}+\cdots+\vec{\lambda}_{n} t_{n} \in X \Rightarrow t_{n}=\nu_{1} t_{1}+\cdots+\nu_{n-1} t_{n-1}+d
$$

Claim 2. Let $t_{1}, \ldots, t_{n}, t_{1}^{\prime}, \ldots, t_{n}^{\prime} \in M$ and $i<n$ be such that $\vec{\lambda}_{1} t_{1}+\cdots+$ $\vec{\lambda}_{n} t_{n}, \vec{\lambda}_{1} t_{1}^{\prime}+\cdots+\vec{\lambda}_{n} t_{n}^{\prime} \in X, t_{i} \neq t_{i}^{\prime}$ and $\forall j \notin\{i, n\}, t_{j}=t_{j}^{\prime}$. Then

$$
\left|s t_{n}\left(t_{n}^{\prime}\right)-s t_{n}\left(t_{n}\right)\right| \leq\left|s t_{i}\left(t_{i}^{\prime}\right)-s t_{i}\left(t_{i}\right)\right| \text {. }
$$

Proof. By (7), we have: $\left|t_{n}^{\prime}-t_{n}\right|<\left|t_{i}^{\prime}-t_{i}\right|$. 
The rest of the argument below resembles the proof of [BO2, Lemma 2.7]. Denote by $\lambda^{*}$ the outer Lebesgue measure on $\mathbb{R}^{n}$. We let

$$
X_{n-1}:=\left\{\vec{\lambda}_{1} t_{1}+\cdots+\vec{\lambda}_{n-1} t_{n-1}: \exists t_{n} \in M, \vec{\lambda}_{1} t_{1}+\cdots+\vec{\lambda}_{n} t_{n} \in X\right\} .
$$

By Claim 2, it is not hard to see that, for every $k \in \mathbb{N}$, st $(X)$ cannot intersect more than $n$ real $\frac{1}{k}$-boxes of dimension $n$ with the same base (a real $\frac{1}{k}$-box of dimension $n-1$ in $\left.\operatorname{st}\left(X_{n-1}\right)\right)$. Since $X \subseteq \Sigma=H^{\Xi}$, we have $\operatorname{st}(X) \subseteq[-\Xi, \Xi]^{n} \subseteq \mathbb{R}^{n}$ and $\operatorname{st}\left(X_{n-1}\right) \subseteq[-\Xi, \Xi]^{n-1} \subseteq \mathbb{R}^{n-1}$. Let $r:=(2 \Xi)^{n-1}=\lambda\left([-\Xi, \Xi]^{n-1}\right)$. Thus, for every $k \in \mathbb{N}$, st $\left(X_{n-1}\right)$ is covered by $r k^{n-1}$ real $\frac{1}{k}$-boxes of dimension $n-1$. Hence for every $k \in \mathbb{N}$, st $(X)$ is covered by $r k^{n-1} n$ real $\frac{1}{k}$-boxes of dimension $n$. Hence, $\lambda^{*}(s t(X)) \leq \frac{1}{k^{n}} r k^{n-1} n$. We have:

$$
\lambda^{*}(s t(X)) \leq \lim _{k \rightarrow \infty}\left[\frac{1}{k^{n}} r k^{n-1} n\right]=0
$$

Thus, $\lambda(s t(X))=0$.

Proof of Theorem 1.6. For every definable $X \subseteq G \subseteq \Sigma$, by (4) and (6) we have:

$$
\mathbf{m}(\pi(X))=r \mathbf{m}^{\prime}(f(\pi(X)))=r \mathbf{m}^{\prime}\left(s t_{G}(X)\right) \leq r \lambda(s t(X)) .
$$

Therefore, by Lemma 4.6, we obtain (1) from Introduction.

Corollary 4.7. (i) $G$ has a unique left (and right) invariant Keisler measure $\mu^{\prime}$, given by: for all $X \in \operatorname{Def}(G), \mu^{\prime}(X)=\mathbf{m}(\pi(X))$.

(ii) For all $X \in \operatorname{Def}(G), \mu^{\prime}(X)>0$ if and only if $X$ is generic.

(iii) Every definable generic subset of $G$ contains a torsion point.

Proof. (i) is by Fact 1.2(ii). For (ii) see Claim 3 in the proof of [HPP, Proposition 9.3], and for (iii) see [HPP, Proposition 10.6].

\section{REFERENCES}

[BO1] A. Berarducci and M. Otero, Intersection theory for o-minimal manifolds, Annals of Pure and Applied Logic 107 (2001), 87-119.

[BO2] A. Berarducci and M. Otero, An additive measure in o-minimal expansions of fields, Quarterly Journal of Mathematics 55 (2004), 411-419.

[BOPP] A. Berarducci, M. Otero, Y. Peterzil and A. Pillay, A descending chain condition for groups definable in o-minimal structures, Annals of Pure and Applied Logic 134 (2005), 303-313.

[BP] Y. Baisalov and B. Poizat, Paires de structures o-minimales, Journal of Symbolic Logic 63 (1998), 570-578.

[vdD] L. van den Dries, Tame topology and o-minimal structures, Cambridge University Press, Cambridge (1998).

[El] P. Eleftheriou, Groups definable in linear o-minimal structures: the non-compact case, to appear in Journal of Symbolic Logic.

[ElSt] P. Eleftheriou and S. Starchenko, Groups definable in ordered vector spaces over ordered division rings, Journal of Symbolic Logic 72 (2007), 1108-1140.

[HPP] E. Hrushovski, Y. Peterzil and A. Pillay, Groups, measures, and the NIP, J. Amer. Math. Soc. 21 (2008), 563-596.

[HP] E. Hrushovski and A. Pillay, On NIP and invariant measures, Preprint (2007).

[Jac] N. Jacobson, Lectures in Abstract Algebra, vol. Volume II - Linear algebra, D. van Nostrand Company, Inc, Princeton, NJ (1953).

[Lang] S. Lang, Algebra, Third Edition, Springer-Verlag, New York, 2002.

[LaPi] D. Lascar and A. Pillay, Hyperimaginaries and automorphism groups, Journal of Symbolic Logic 66 (2001), 127-143. 
[LoPe] J. Loveys and Y. Peterzil, Linear o-minimal structures, Israel Journal of Mathematics 81 (1993), 1-30.

[Ons] A. Onshuus, Groups definable in $\langle\mathbb{Q},+,<\rangle$, Preprint (2005).

[Pet] Y. Peterzil, Returning to semi-bounded sets, to appear in Journal of Symbolic Logic.

[PePi] Y. Peterzil and A. Pillay, Generic sets in definably compact groups, Fundamenta Mathematicae (2) 193 (2007), 153-170.

[PeSt] Y. Peterzil and S. Starchenko, Definable homomorphisms of abelian groups in o-minimal structures, Annals of Pure and Applied Logic 101 (2000), 1-27.

[PeS] Y. Peterzil and C. Steinhorn, Definable compactness and definable subgroups of ominimal groups, J. London Math. Soc. (2) 69 (1999), 769-786.

[Pi1] A. Pillay, On groups and fields definable in o-minimal structures, J. Pure Appl. Algebra 53 (1988), 239-255.

[Pi2] A. Pillay, Type definability, compact Lie groups, and o-minimality, J. of Math. Logic, 4 (2004), 147-162.

Cmaf, Universidade de Lisboa, Av. Prof. Gama Pinto 2, 1649-003 Lisboa, Portugal E-mail address: pelefthe@ptmat.fc.ul.pt 